\title{
Association between acute phase reactants, interleukin-6, tumor necrosis factor-a, and disease activity in Takayasu's arteritis patients
}

Jing $\mathrm{Li}^{1+}{ }^{+}$, Yahong Wang ${ }^{2+}$, Yanhong Wang ${ }^{3+}$, Ying Wang ${ }^{2}$, Yunjiao Yang ${ }^{1}$, Jiuliang Zhao ${ }^{1}$, Mengtao $\mathrm{Li}^{1}$, Xinping $\operatorname{Tian}^{1 *}$ (1) and Xiaofeng Zeng ${ }^{1 *}$ (i)

\begin{abstract}
Background: To investigate the association between blood biomarkers and disease activity of Takayasu's arteritis (TAK) in a follow-up cohort.

Methods: Disease activity was assessed by clinical manifestations and repeated vascular Doppler examinations. The association between erythrocyte sedimentation rate (ESR), serum levels of high-sensitive C-reactive protein (hsCRP), interleukin-6(IL-6), and tumor necrosis factor-a (TNFa) and disease activity were analyzed by logistic regression and survival analysis. Kaplan-Meier method was used to estimate the cumulative remission rate curve, log-rank tests for group comparison, and Cox regression for estimating hazard ratios of these parameters for disease activity.

Results: 428 patients were included. 188 patients were in active disease, and 240 patients were in inactive disease at baseline. Elevation of ESR, hsCRP, and IL-6 were associated with active disease at baseline and during follow-up. Cox regression and Kaplan-Meier analysis showed that lower possibility and longer time to remission were associated with elevated ESR (hazard ratio $[H R]=0.32$, 80 vs 33 weeks, $p<0.001$ ), hsCRP (HR=0.45, 70 vs 31 weeks, $p<0.001$ ), and IL-6 (HR= $0.54,66$ vs 34 weeks, $p<0.01$ ) in patients with active disease at baseline, while higher risk and shorter time for relapse were associated with elevated ESR (HR=2.1, 59 vs 111 weeks, $p<0.001)$, hsCRP (HR=2.1, 79 vs 113 weeks, $p<0.001)$, L-6 (HR= $2.5,64$ vs 117 weeks, $p<0.001)$, and TNFa ( $H R=2.7,65$ vs 114 weeks, $p<0.001)$ in patients with inactive disease at baseline.

Conclusions: Elevated ESR, CRP, and IL-6 are associated with active disease, lower possibility, and longer time to achieve disease remission. Elevation of any among ESR, CRP, IL-6, and TNFa is associated with high risk and short time for relapse during follow-up.
\end{abstract}

Keywords: Takayasu arteritis, Angiographic examination, Disease activity, Acute phase reactants, Interleukin-6, Tumor necrosis factor-a

*Correspondence: tianxp6@126.com; xiaofeng.zeng@cstar.org.cn

${ }^{+}$Jing Li, Yahong Wang and Yanhong Wang contributed equally to this work.

'Department of Rheumatology, Peking Union Medical College Hospital,

Peking Union Medical College and Chinese Academy of Medical Sciences,

National Clinical Research Center for Dermatologic and Immunologic

Diseases (NCRC-DID), Key Laboratory of Rheumatology and Clinical

Immunology, Ministry of Education, Beijing 100730, China

Full list of author information is available at the end of the article

C C The Author(s). 2020 Open Access This article is licensed under a Creative Commons Attribution 4.0 International License, which permits use, sharing, adaptation, distribution and reproduction in any medium or format, as long as you give appropriate credit to the original author(s) and the source, provide a link to the Creative Commons licence, and indicate if changes were made. The images or other third party material in this article are included in the article's Creative Commons licence, unless indicated otherwise in a credit line to the material. If material is not included in the article's Creative Commons licence and your intended use is not permitted by statutory regulation or exceeds the permitted use, you will need to obtain permission directly from the copyright holder. To view a copy of this licence, visit http://creativecommons.org/licenses/by/4.0/ The Creative Commons Public Domain Dedication waiver (http://creativecommons.org/publicdomain/zero/1.0/) applies to the data made available in this article, unless otherwise stated in a credit line to the data. 


\section{Background}

Takayasu's arteritis (TAK) is an uncommon systemic vasculitis that involves aorta and its major branches [1]. The assessment of disease activity in TAK patients is very challenging due to its complicated clinical features and lack of reliable biomarkers. Erythrocyte sedimentation rate (ESR) and C-reactive protein (CRP) were widely used acute phase reactants in monitoring disease activity of patients with TAK. However, ESR and CRP are not reliable indicators because the disease could progress in patients with normal ESR and CRP levels [2].

Interleukin-6 (IL-6) and tumor necrosis factor- $\alpha$ (TNF $\alpha$ ) have been shown to be involved in the pathogenesis of TAK $[3,4]$. Both of them were over-expressed in the diseased aorta and its branches [5]. Therefore, the elevated level of TNF $\alpha$ and IL- 6 may be associated with active disease.

Aorta and its major branches are targets of TAK. Wall thickness and mural stenosis are the typical changes in TAK [6]. The progression of or new onset of wall thickness and stenosis are regarded as the gold indicator for active disease [7]. Therefore, if blood biomarkers that are correlated with vascular image progression and clinical active disease manifestations could be found, it would greatly help rheumatologists to make appropriate medical decisions and improve the long-term prognosis of TAK patients. Based on this hypothesis, we conducted this prospective study aimed to explore the association between blood biomarkers with disease activity of TAK, taking vascular changes as the gold standard.

\section{Patients and methods}

\section{Patient population}

Chinese Registry for Systemic Vasculitis (CRSV), based on the CRDC (Chinese Rheumatism Data Center) platform, was developed to collect the clinical information and prognosis data of Chinese patients with systemic vasculitis. The CRSV registry was initiated in July 2013. So far, 118 member centers participated in the registry. Up to April 30, 2019, 1150 patients with TAK were registered in CRSV. All the registered patients must fulfill the 1990 American College of Rheumatology (ACR) classification criteria for Takayasu's arteritis [8]. Among them, 588 patients were registered and followedup in Peking Union Medical College Hospital (PUMCH). At the time of registration, computed tomography angiography (CTA) was performed in all patients to confirm the diagnosis and evaluate the extent of artery involvement.

Patients in this study were followed every 1 and 3 to 6 months according to patients' disease activity status. At each visit, ESR, blood hs-CRP, TNFo, and IL-6 levels were tested. Disease activity was comprehensively assessed based on clinical presentations and Doppler examination results at each visit. Repeated vascular
Doppler examinations were performed every 3 to 6 months according to the patient's disease activity status. Patients with active disease in the first visit were followed-up 1-3 months later. Patients were followed every 6 months if they were assessed to be in inactive disease at the first visit. All patients had vascular Doppler imaging examination at baseline and every followup visit.

This study was approved by the Institutional Review Board of Peking Union Medical College Hospital (S-478), Beijing, China. Written informed consent was obtained from all participants, and the study was performed in accordance with the Declaration of Helsinki. Personal information was protected and kept anonymous in CRSV database.

\section{Clinical data}

The demographic data and medical histories were collected when patients were registered in CRSV. The clinical manifestations, Birmingham Vasculitis Activity Score (BVAS), Vasculitis Damage Index (VDI) score, laboratory tests (including ESR, serum levels of hsCRP, IL-6, and TNF $\alpha$ ), imaging findings (including serial vascular Doppler imaging examination, CTA, etc.), and treatment regimen were recorded at each visit. Outcomes, complications, and adverse events related to medications were recorded if occurred.

Serum levels of IL- 6 and TNF-alpha were detected with Siemens LK6P1 and LKNF1 assay kit by IMMULITE/IMMULITE 1000 system at each visit in our center.

Repeated vascular Doppler of carotid arteries, vertebral arteries, subclavian arteries, axillary artery, ulnar and radial artery, abdominal aorta, renal arteries, celiac arteries, and mesangial arteries were performed and compared with former results to identify changes. Since the reconstruction of the vessel wall is a continued process, the changes of vascular images might be found later than that of clinical disease flare. The information of image results was stored in the electronic clinical information system of our center.

\section{Evaluation of disease activity}

Disease activity of patient with TAK was evaluated by 2 senior rheumatologists who have more than 5 years of experiences in taking care of TAK patients. They were responsible for judging the disease activity based on comprehensive assessment of clinical manifestations and serial vascular Doppler imaging findings at each visit. In this study, all vascular examinations were performed by 2 vascular Doppler specialists who had ever been trained for large vessel Doppler examination. They were blind to the lab test results of patients. The "image active disease" was defined as new occurrence of stenosis or occlusion or dilatation (including aneurysm), worsen mural 
thickness or stenosis. The definition of active disease was also defined as the presence of the following symptoms or signs after other causes were excluded ("clinical active disease"): fever, weight loss, fatigue and/or arthralgia/arthritis/myalgia, new onset or aggravated symptoms of ischemia (including limb claudication, stroke, dizziness, syncope, severe abdominal pain, myocardial infarction, or angina), acute visual symptoms such as amaurosis fugax or diplopia, new onset hypertension, new onset of vessel bruit, new loss of pulses, carotidynia or tenderness of vessels, and other conditions judged by the 2 senior rheumatologists that increase the dosage of corticosteroid and/or necessary addition of immunosuppressive drugs. Patients with any among "clinical active disease", "image active disease", or judged by the rheumatologist as active were defined as "active disease." If the patient had none of the clinical manifestations for active disease and did not have active image presentations, or judged by the rheumatologist as in inactive disease, then the patient was defined as "inactive disease." Flare was defined when the patient's disease status changed from "inactive disease" to "active disease," and remission was defined when the patient's disease status changed from "active disease" to "inactive disease."

\section{Visits during follow-up}

The visit when all the above blood parameters were tested and data were collected from serial vascular Doppler imaging examination for future comparison was defined as the baseline visit. Then, patients were followed-up at 1and 3 to 6 -month intervals depending on the patient's situation and disease activity status was evaluated by the 2 rheumatologists. At each follow-up visit, comprehensive history taking and physical examination were done, all the parameters were tested, and vascular Doppler imaging examination was repeated every 3 to 6 months.

\section{Statistical analysis}

Continuous variables were expressed as the mean \pm standard deviation for the data in a normal distribution and median (quantile 1, quantile 3) for the non-normal distributed data. Kolmogorov-Smirnov test was used to test data normality. If normal distribution was satisfied, $t$ test was used to compare between groups. Categorical variables were expressed as absolute numbers and percentages, and chi-squared test was used to compare between groups.

In our analysis, serum level of TNF $\alpha$ was categorized into normal and abnormal groups by the upper limit of normal range in healthy population $(\leq 8.1 \mathrm{pg} / \mathrm{ml})$, so was serum level of IL-6 (upper limit of normal range $\leq 5.9$ $\mathrm{pg} / \mathrm{ml}$ ), hsCRP (upper limit of normal range $\leq 8 \mathrm{mg} / \mathrm{L}$ ), and ESR (upper limit of normal range $\leq 20 \mathrm{~mm} / 1$ st hour).
The univariate logistic regression analysis was performed to investigate the association between these parameters (ESR, hsCRP, IL-6, and TNF $\alpha$ ) and disease activity at baseline. Furthermore, sensitivity, specificity, and the 95\% confidence intervals were calculated. For follow-up data, univariate Cox regression model was used to explore the association of ESR, hsCRP, IL-6, and TNF $\alpha$ with disease activity in the follow-up visits. Positive predictive values and negative predictive values, as well as the $95 \%$ confidence intervals were also represented. Kaplan-Meier method was conducted to describe the curve of cumulative remission rate, and log-rank tests were used to compare cumulative remission curves.

For patients with active disease at baseline but turned inactive during follow-up, the follow-up time in survival analysis was the duration from the date of baseline visit to the date of visit when inactive disease was first achieved. For those patients who were still in active state in later follow-up visits, their follow-up time was defined from the date of baseline visit to the end of the study.

For patients who were in inactive disease at baseline but changed to active diseases during follow-up, their follow-up duration was defined from the date of baseline visit to the date of visit for first relapse. For patients who remained in inactive during follow-up, their follow-up duration was defined from the date of baseline visit to the end of the study.

A two-sided $p$ value less than 0.05 was considered to be statistically significant, and the odds ratio (OR) or hazard ratio (HR) with a $95 \%$ confidence interval $(\mathrm{CI})$ were also calculated. Analysis was performed with the SAS software (version 9.0, SAS Institute, Cary, NC, USA).

\section{Results}

Demographic data, clinical features, and laboratory findings of patients

428 patients were included in this study prospectively from PUMCH. Based on the definition described above, 188 patients were defined as with "active disease" and 240 patients were defined as with "inactive disease" at baseline (Fig. 1).

Demographic data, laboratory test results, and repeated Doppler image results of these patients at baseline were summarized in Table 1. There was no significant difference between these two groups in age $(p=0.26)$, gender $(p=0.63)$, and disease durations $(p=0.51)$. In active group at baseline, the median follow-up time (33 months) was shorter than in inactive group $(64$ months $)(p<0.001)$ at baseline (Table 1).

Comparisons between active and inactive groups at baseline showed significant differences in the levels of ESR, hsCRP, IL-6, and TNF $\alpha$. While comparing the proportions of patients with elevated level of these four parameters, no significant difference was found between 
the two groups for TNF $\alpha(p=0.19)$ only, while there was a significant difference in the other three parameters (Table 1).

There was no significant difference in clinical manifestations between these two groups, as well as in BVAS scores and the VDI scores. The results of comparison between these two groups were summarized in Supplementary Table-S1.

\section{Association between laboratory findings with disease activity at baseline}

Elevated level of ESR (OR =5.34, 95\%CI $3.27 \sim 8.72)$, hsCRP $(\mathrm{OR}=13.60,95 \% \mathrm{CI} 8.18 \sim 22.62)$, and IL-6 (OR = $7.75,95 \%$ CI $4.17 \sim 14.42$ ) of 428 patients were found to be associated with active disease activity at baseline in univariate logistic regression. But elevated level of TNF $\alpha$ $(\mathrm{OR}=1.42$, 95\%CI $0.85 \sim 2.37)$ was not associated with active disease at baseline (Table 2). After excluding patients treated with TNF $\alpha$ inhibitors or IL- 6 inhibitor and patients in pregnancy, the results remained the same (Supplementary Table-S2).

Furthermore, sensitivity and specificity were also be reported in Table 2 . The specificity was $88.1 \%$ for ESR, $88.7 \%$ for hsCRP, $85.8 \%$ for IL-6, and $60.3 \%$ for TNF $\alpha$. And the sensitivity was $41.9 \%$ for ESR, $63.5 \%$ for hsCRP, $56.1 \%$ for IL-6, and $48.2 \%$ for TNFa.

\section{Survival analysis of ESR, hsCRP, IL-6, and TNFa with disease activity during follow-up}

In this study, 152 patients of the active group and 202 patients of the inactive group at baseline were followedup and included in the survival analysis (Fig. 1). Among 152 patients of the active group, 120 patients (79.0\%) achieved inactive disease in the follow-up visit, and the median time to achieve inactive disease was 35 weeks (95\% CI 31-40 weeks). Moreover, in 202 patients of the inactive group, 106 patients (52.5\%) were found to relapse in the follow-up visit. The median time to relapse was 94 weeks (95\%CI 75-109 weeks).

As showed in Fig. 2, elevated levels of ESR (2A, 80 vs 33 weeks), hsCRP (2B, 70 vs 31 weeks), and IL-6 (2C, 66 vs 34 weeks) at baseline were associated with longer time to achieve remission in those patients with active disease at baseline $(p<0.01)$ (Fig. 2, Supplementary Table-S3). Elevated levels of ESR (3A, 59 vs 111 weeks), hsCRP (3B, 79 vs 113 weeks), IL-6 (3C, 64 vs 117 weeks), and TNF $\alpha$ (3D, 65 vs 114 weeks) were associated with shorter time to relapse in those patients with inactive disease at baseline $(p<0.001)$. (Fig. 3, Supplementary Table-S4).

Cox regression also revealed that elevated levels of ESR (HR: 0.32, 95\%CI 0.18-0.58), hsCRP (HR: 0.45, 95\%CI 0.30-0.68), and IL-6 (HR: 0.54, 95\%CI 0.34-0.84) were associated with lower possibility to achieve an inactive state in patients with active group at baseline. In addition, the elevated level of ESR (HR: 2.11, 95\%CI 1.41-3.16), hs-CRP (HR: 2.13, 95\%CI 1.42-3.20), IL-6 (HR: 2.50, 95\%CI 1.60-3.91), and TNF $\alpha$ (HR: 2.65, 95\%CI 1.69-4.15) were found to be associated with higher risk for disease relapse in patients of the inactive group at baseline (Table 3 ).

The positive predictive value and negative predictive value are presented in Table 3 . In active patients at baseline, compared to the positive predictive values, higher negative predictive values were found to be $86.2 \%$ for ESR, 91.3\% for hsCRP, $87.8 \%$ for IL-6, and 76.7\% for TNF- $\alpha$. On the contrary, in inactive patients at baseline, higher positive predictive value was found to be $80.9 \%$ for ESR, 82.2\% for hsCRP, 78.1\% for IL-6, and $65.2 \%$ for TNF- $\alpha$.

Repeated vascular Doppler image results of patients at follow-up visits are shown in Table 4.

\section{Discussion}

TAK is characterized by relapse and remission in the course of the disease. The goal of the management of

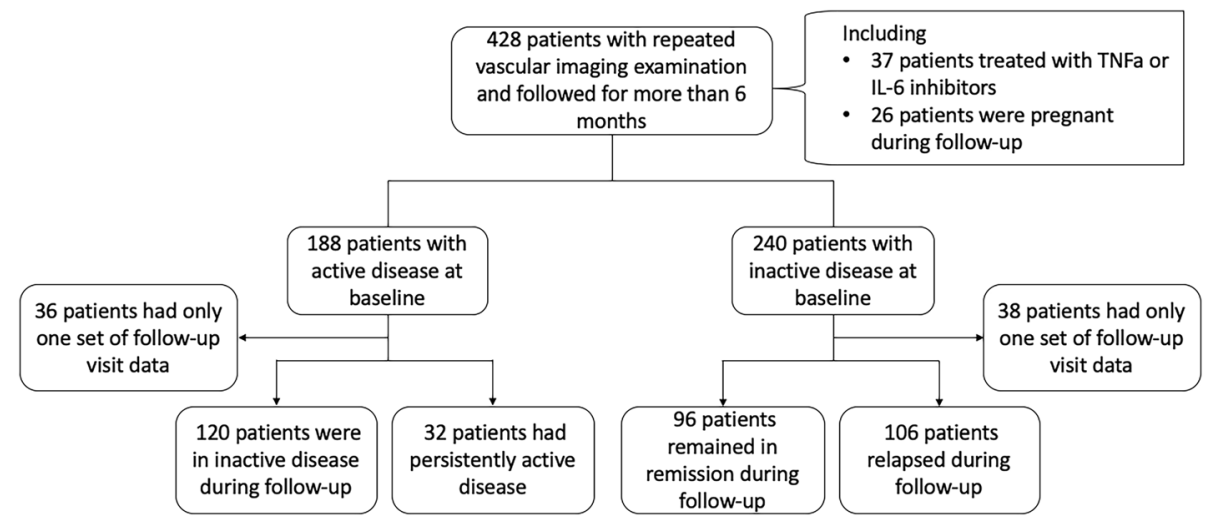

Fig. 1 The flow chart of the study and data collection. The patients with Takayasu's arteritis in this study were all from the Chinese Registry of Systemic Vasculitis (CRSV). Clinical data of 428 patients with repeated vascular imaging examination and followed more than 6 months were collected 
Table 1 The demographic data, BVAS and VDI scores, laboratory test results, and Doppler image results of 428 patients with Takayasu's arteritis at baseline

\begin{tabular}{|c|c|c|c|c|}
\hline & Total $(n=428)$ & Active group $(n=188)$ & Inactive group $(n=240)$ & $p$ value \\
\hline Age, years & $31.8 \pm 9.5$ & $31.8 \pm 9.9$ & $31.8 \pm 9.1$ & 0.26 \\
\hline Female, $n(\%)$ & $393(91.8)$ & $174(92.6)$ & $219(91.3)$ & 0.63 \\
\hline Disease duration, months (Q1, Q3) & $53(29,99)$ & $52(29,107)$ & $54(31,96)$ & 0.51 \\
\hline Median follow-up time, months (Q1, Q3) & $49(28,85)$ & $33(25,61)$ & $64(34,98)$ & $<0.001$ \\
\hline BVAS scores & $6.7 \pm 3.7$ & $7.0 \pm 3.7$ & $6.4 \pm 3.7$ & 0.10 \\
\hline VDI scores & $2.7 \pm 1.8$ & $2.8 \pm 2.0$ & $2.6 \pm 1.6$ & 0.28 \\
\hline \multicolumn{5}{|l|}{ Laboratory test results } \\
\hline ESR, mm/1st hour & $17.1 \pm 19.6$ & $25.6 \pm 25.1$ & $10.4 \pm 9.4$ & $<0.001$ \\
\hline ESR > 20 mm/1st hour, $n(\%)$ & $106 / 421^{*}(25.2)$ & $78 / 186^{*}(41.9)$ & $28 / 235^{*}(11.9)$ & $<0.001$ \\
\hline hsCRP, mg/L & $13.2 \pm 26.7$ & $25.6 \pm 36.2$ & $3.4 \pm 5.5$ & $<0.001$ \\
\hline hsCRP > 8 mg/L, n (\%) & $141 / 410^{*}(34.4)$ & $115 / 181^{*}(63.5)$ & $26 / 229^{*}(11.4)$ & $<0.001$ \\
\hline $\mathrm{IL}-6, \mathrm{pg} / \mathrm{ml}$ & $13.9 \pm 40.0$ & $21.2 \pm 42.3$ & $7.4 \pm 36.7$ & 0.003 \\
\hline IL-6 > 5.9 pg/ml, n (\%) & $82 / 241^{*}(34.0)$ & $64 / 114^{*}(56.1)$ & $18 / 127^{*}(14.2)$ & $<0.001$ \\
\hline TNFa, pg/ml & $16.3 \pm 34.2$ & $19.9 \pm 44.3$ & $13.0 \pm 21.3$ & 0.02 \\
\hline TNFa $>8.1 \mathrm{pg} / \mathrm{ml}, n(\%)$ & $104 / 238^{*}(43.7)$ & $54 / 112^{*}(48.2)$ & $50 / 126^{*}(39.7)$ & 0.19 \\
\hline \multicolumn{5}{|l|}{$\begin{array}{l}\text { Repeated vascular Doppler image results } \\
\text { compared to former results }\end{array}$} \\
\hline Stable & $175(40.1 \%)$ & $27(14.4 \%)$ & $148(61.7 \%)$ & \\
\hline Improved & $15(3.5 \%)$ & $2(1.1 \%)$ & $13(5.4 \%)$ & \\
\hline Progressed & $238(55.6 \%)$ & $159(84.6 \%)$ & 79 (32.9\%) & \\
\hline
\end{tabular}

Abbreviation: ESR erythrocyte sedimentation rate, hsCRP high sensitive C-reactive protein, IL-6 interleukin-6, TNFa tumor necrosis factor-a

\#Among the follow-up patients

${ }^{*}$ Actually detected

TAK is to induce the disease into remission and maintain the disease in persistent inactive condition in order to preserve blood supply and improve the long-term outcome. However, assessing disease activity is difficult and very challenging. Although a number of assessment instruments or biomarkers had been developed to assess disease activity, none of them were reliable or easy to apply in clinical practice. Birmingham Vasculitis Activity Score (BVAS) [9], physician global assessment (PGA) [10], Disease Extent Index-Takayasu (DEI.Tak) [11], and Indian Takayasu Activity Score (ITAS) were the most commonly used instruments [12]. BVAS is not well suitable for disease activity assessment for large vessel vasculitis, so it is not routinely used for disease activity evaluation for
TAK in clinical practice. The ITAS was derived from the DEI.Tak. These two instruments only include clinical symptoms and signs but do not include imaging findings and acute phase reactants $[11,12]$. Though the ITAS had shown good correlation with PGA in an Indian center [12], it is time-consuming and not easy to apply in daily clinical practice.

ESR and CRP are the most commonly used biomarkers for the assessment of disease activity of TAK. The sensitivity and specificity of ESR for active TAK are $72 \%$ and $56 \%$, respectively [13]. Although CRP has a sensitivity of $71.4 \%$ and specificity of $100 \%$ for active disease in a study [14], CRP may elevate nonspecifically in tissue inflammation and infection, so it may not accurately reflect the disease

Table 2 Association between ESR, hsCRP, IL-6 and TNFa with disease activity at baseline in logistic regression. Results of univariate logistic regression analysis were shown in this table, which analyzed the association between ESR, hsCRP, IL-6 and TNFa with disease activity at baseline in 428 patients

\begin{tabular}{lllll}
\hline & Odds ratio $\mathbf{( 9 5 \% ~ C l )}$ & $\boldsymbol{p}$ value & Sensitivity (95\% Cl) & Specificity (95\% Cl) \\
\hline ESR $(>20 \mathrm{~mm} / 1$ st hour) & $5.34(3.27 \sim 8.72)$ & $<0.001$ & $41.9 \%(34.8 \%-49.4 \%)$ & $88.1 \%(83.2 \%-91.9 \%)$ \\
hsCRP $(>8 \mathrm{mg} / \mathrm{L})$ & $13.60(8.18 \sim 22.62)$ & $<0.001$ & $63.5 \%(56.1 \%-70.6 \%)$ & $88.7 \%(83.8 \%-92.5 \%)$ \\
$\mathrm{IL}-6(>5.9 \mathrm{pg} / \mathrm{ml})$ & $7.75(4.17 \sim 14.42)$ & $<0.001$ & $56.1 \%(46.5 \%-65.4 \%)$ & $85.8 \%(78.5 \%-91.4 \%)$ \\
TNFa $>8.1 \mathrm{pg} / \mathrm{ml})$ & $1.42(0.85 \sim 2.37)$ & 0.19 & $48.2 \%(38.7 \%-57.9 \%)$ & $60.3 \%(51.2 \%-68.9 \%)$ \\
\hline
\end{tabular}



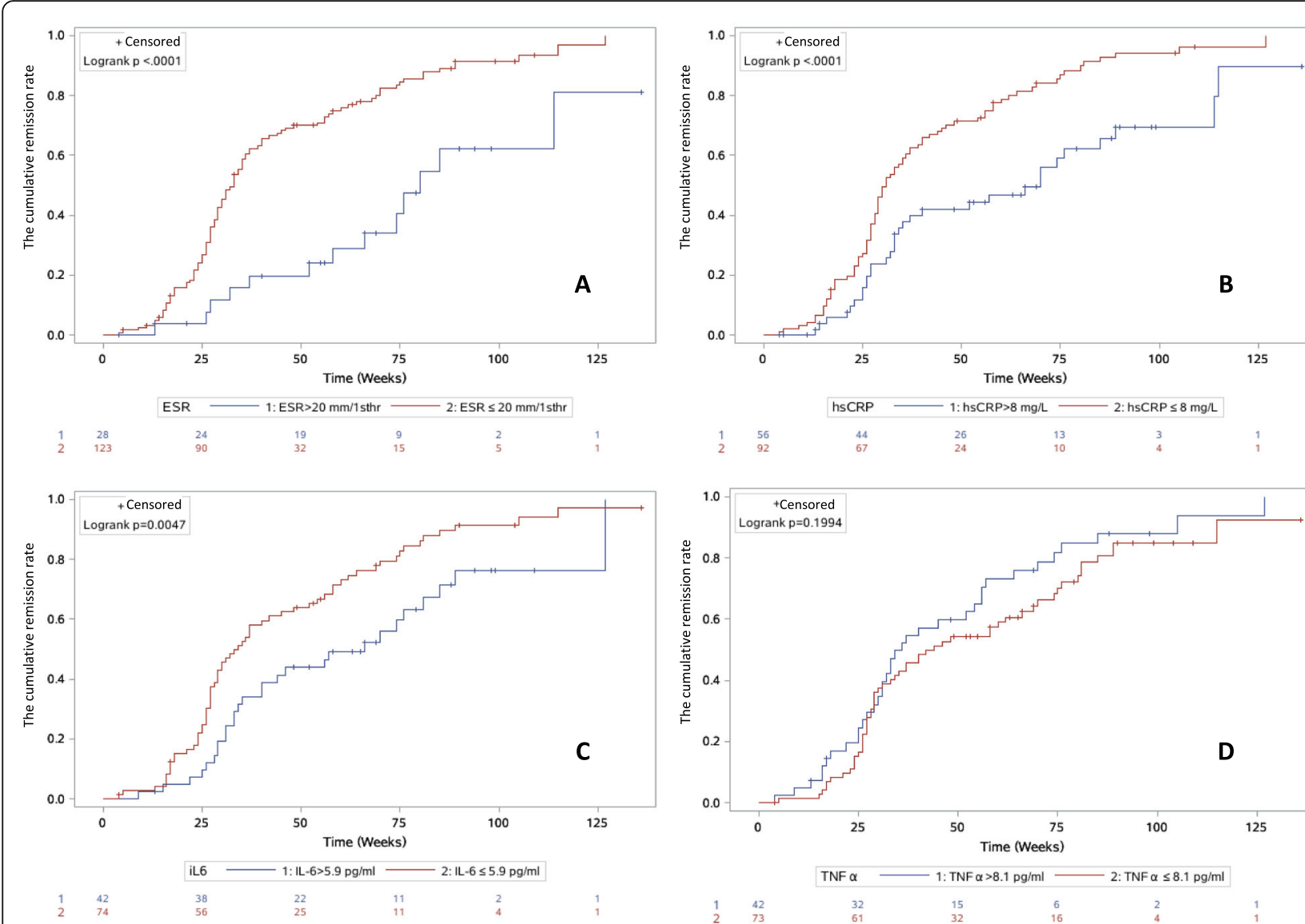

Fig. 2 The cumulative remission rate curves by using Kaplan-Meier analysis for the active group at baseline. This figure demonstrated the cumulative remission rate curves by using Kaplan-Meier analysis of 152 patients in active group at baseline, with further follow-up data. Elevated levels of ESR (2A, 80 vs 33 weeks, $p<0.0001$ ), hsCRP ( $2 B, 70$ vs 31 weeks, $p<0.0001)$, and IL-6 (2C, 66 vs 34 weeks, $p<0.01)$ at baseline were associated with longer time to achieve remission in those patients with active disease at baseline

status. High sensitive CRP (hs-CRP) is developed to detect CRP in a more sensitive way, but had the same limitations as CRP. Furthermore, TAK could relapse or progress in the absence of elevated CRP and ESR. Many biomarkers had been investigated to assess disease activity of TAK in addition to ESR and CRP. Levels of serum IL- 6 and TNF $\alpha$ were found to be elevated in patients with TAK, and IL-6 levels were higher in those with active disease [15]. However, so far, no study has been reported to investigate the association between these parameters with disease activity during follow-up study or to take vascular image changes as the major evidence into consideration for disease activity evaluation. In this study, we explored the role of ESR, hsCRP, IL-6, and TNF $\alpha$ in the assessment of disease activity in a large follow-up cohort of Chinese TAK patients.

In our study, patients were followed-up at 3- to 6month interval regularly. At each visit, symptoms and signs were recorded and examined, vascular Doppler imaging examinations were performed and levels of acute phase reactants, and TNF $\alpha$ and IL- 6 were tested. All these enabled us to evaluate patient's disease activity more comprehensively and accurately than basing our evaluation solely on the acute phase reactants levels.

In this study, the inflammatory parameters, including the elevation of ESR, hsCRP, and IL-6, were found to be associated with active disease of TAK by logistic regression. This result was consistent with the study by Tamura and co-researchers [15]. Furthermore, in this study, we found that ESR, hsCRP, TNF $\alpha$, and IL- 6 were associated with active disease during follow-up by survival analysis, both in Cox regression and Kaplan-Meier models. This suggested that test for these 4 parameters might provide evidence for rheumatologists to determine disease activity of TAK patients.

Our study also showed that elevated ESR, hs-CRP, IL6 , and TNFo were independently associated with disease relapse during follow-up. In cohort study of Comarmond and co-researchers, elevated level of ESR and CRP was associated with the relapse during follow-up [16]. Our study not only confirmed their results in a much larger patient population, but also showed that ESR, hsCRP, TNF $\alpha$, and IL-6 were associated with a higher risk for 

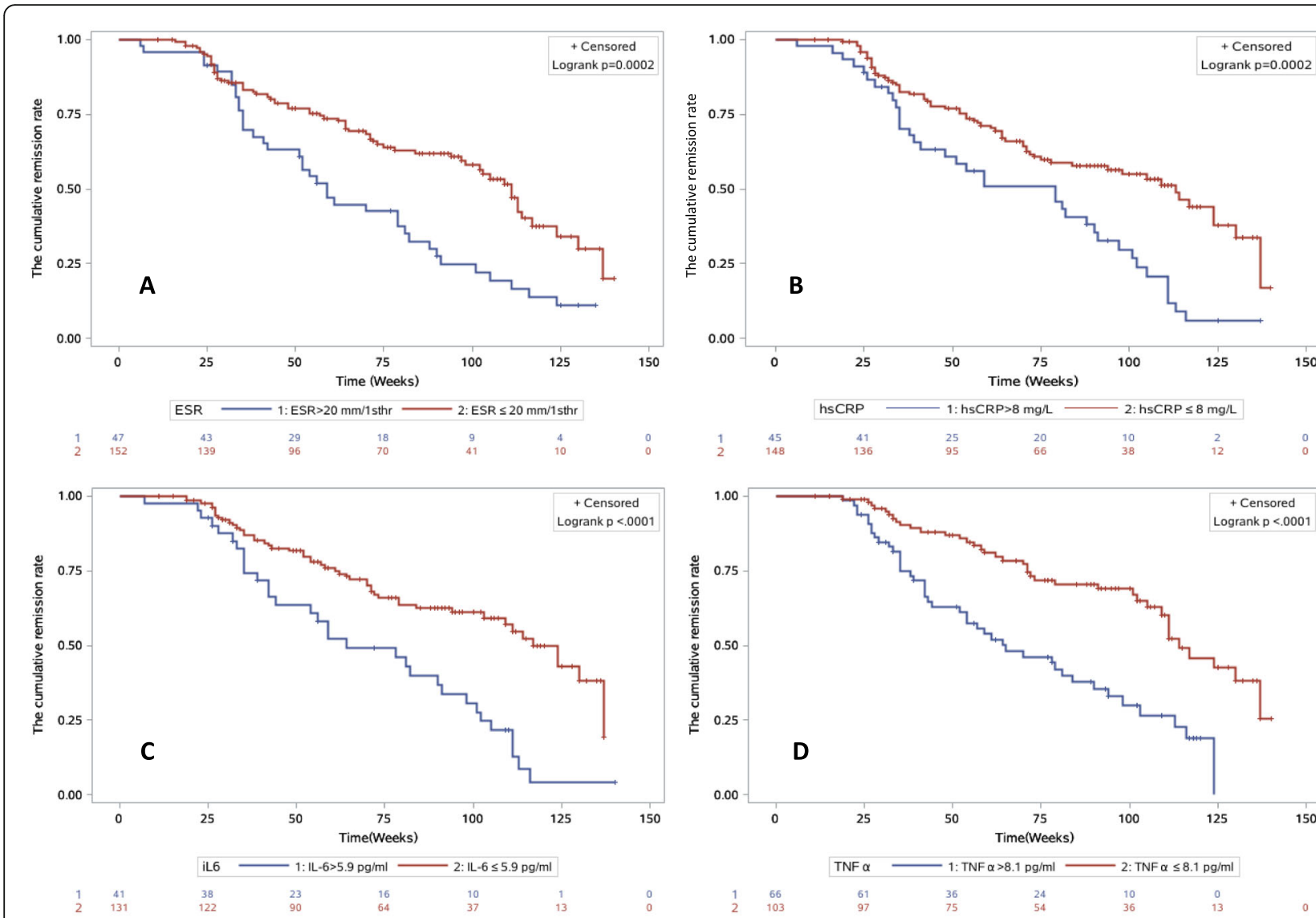

Fig. 3 The cumulative remission rate curves by using Kaplan-Meier analysis for the inactive group at baseline. This figure demonstrated the cumulative remission rate curves by using Kaplan-Meier analysis of 202 patients in inactive group at baseline with further follow-up data. Elevated levels of ESR (3A, 59 vs 111 weeks, $p<0.001$ ), hsCRP (3B, 79 vs 113 weeks, $p<0.001$ ), IL-6 (3C, 64 vs 117 weeks, $p<0.0001$ ), and TNFa (3D, 65 vs 114 weeks, $p<0.0001)$ were associated with shorter time to relapse in those patients with inactive disease at baseline

relapse during follow-up when compared with patients with normal levels of ESR, hsCRP, TNFa, and IL-6. This is very helpful in patients whose seral ESR and/or hsCRP level were not elevated. Our results also demonstrated that patients who were currently inactive, with elevated ESR, CRP, IL-6, and TNF $\alpha$ would relapse in a shorter time during follow-up compared to patients with normal ESR, hs-CRP, IL-6, and TNFa. Based on this result, we might conclude that these parameters not only provided clues for active disease, but also predicted upcoming relapse in near future.

The elevation of IL-6 was not only associated with active disease of TAK at baseline, but also associated with active disease during follow-up, including longer time to achieve remission in patients of active group at baseline and shorter time to relapse in inactive group at baseline. While elevation of TNF $\alpha$ was found to be associated with shorter time to relapse in patients with inactive disease at baseline, it is not associated with longer time to achieve inactive state. Previous studies had shown that these two indices are elevated in active TAK patients [15] as compared to healthy controls or patients in remission [17], but did not investigate their association with relapse during follow-up. Results of this study suggested that detection of TNF $\alpha$ and IL- 6 was not only useful to help evaluate disease activity in the present visit, but also helpful to identify patients at high risk of relapse.

There are some limitations in our study. First, the treatment was not considered as a control variable in the models. For patients with inactive disease at baseline, the treatment would be kept the same or adjusted in order to get persistent remission even though the dosage of glucocorticosteroids was tapered to minimize the side effects of the drug. Therefore, the effect of drugs was minimized in this group of patients. However, in patients with active disease at baseline, the dosage of glucocorticosteroid would be increased or the DMARDs were added to induce remission. The adjustment of therapy was individualized based on treatment history and disease severity, which was difficult to standardize. Therefore, the impact of drugs could not be put into our 
Table 3 Cox regression analysis on the association of laboratory results with disease activity in follow-up visits. Results of Cox regression analysis were shown in this table, which analyzed the association of ESR, hsCRP, IL-6, and TNFa with inactive disease or relapse in follow-up visits among 354 Takayasu's arteritis patients who were followed-up

\begin{tabular}{|c|c|c|c|c|c|c|c|}
\hline & & \multicolumn{2}{|c|}{$\begin{array}{l}\text { Disease activity in } \\
\text { follow-up visits }\end{array}$} & \multirow[t]{2}{*}{$p$ value } & \multirow[t]{2}{*}{ HR $(95 \% \mathrm{Cl})$} & \multirow{2}{*}{$\begin{array}{l}\text { Positive predictive value } \\
(95 \% \mathrm{Cl})\end{array}$} & \multirow{2}{*}{$\begin{array}{l}\text { Negative predictive value } \\
(95 \% \mathrm{Cl})\end{array}$} \\
\hline & & Inactive & Active & & & & \\
\hline \multicolumn{8}{|c|}{152 patients in active group at baseline } \\
\hline \multicolumn{2}{|c|}{ Number of patients } & 120 & 32 & & & & \\
\hline \multirow[t]{2}{*}{ ESR } & $(\leq 20 \mathrm{~mm} / 1 \mathrm{st}$ hour $)$ & $106(86.2 \%)$ & $17(13.8 \%)$ & $<0.001$ & 1.00 & $53.6 \%(33.9-72.5 \%)$ & $86.2 \%(78.8-91.7 \%)$ \\
\hline & (> $20 \mathrm{~mm} / 1$ st hour) & $13(46.4 \%)$ & $15(53.6 \%)$ & & $0.32(0.18-0.58)$ & & \\
\hline \multirow[t]{2}{*}{ hsCRP } & $(\leq 8 \mathrm{mg} / \mathrm{L})$ & $84(91.3 \%)$ & $8(8.7 \%)$ & $<0.001$ & 1.00 & $42.7 \%(29.7-56.8 \%)$ & $91.3 \%(83.6-96.2 \%)$ \\
\hline & (> $8 \mathrm{mg} / \mathrm{L})$ & $32(57.1 \%)$ & $24(42.9 \%)$ & & $0.45(0.30-0.68)$ & & \\
\hline \multirow[t]{2}{*}{ IL-6 } & $(\leq 5.9 \mathrm{pg} / \mathrm{ml})$ & $65(87.8 \%)$ & $9(12.2 \%)$ & 0.006 & 1.00 & $33.3 \%(19.6-49.6 \%)$ & $87.8 \%(78.2-94.3 \%)$ \\
\hline & (> $5.9 \mathrm{pg} / \mathrm{ml})$ & $28(66.7 \%)$ & $14(33.3 \%)$ & & $0.54(0.34-0.84)$ & & \\
\hline \multirow[t]{2}{*}{ TNFa } & $(\leq 8.1 \mathrm{pg} / \mathrm{ml})$ & $56(76.7 \%)$ & $17(23.3 \%)$ & 0.25 & 1.00 & $14.3 \%(5.4-28.5 \%)$ & $76.7 \%(65.4-85.8 \%)$ \\
\hline & $(>8.1 \mathrm{pg} / \mathrm{ml})$ & $36(85.7 \%)$ & $6(14.3 \%)$ & & $1.31(0.86-2.00)$ & & \\
\hline \multicolumn{8}{|c|}{202 patients in inactive group at baseline } \\
\hline \multicolumn{2}{|c|}{ Number of patients } & 96 & 106 & & & & \\
\hline \multirow[t]{2}{*}{ ESR } & $\left(\leq 20 \mathrm{~mm} / 1^{\mathrm{st}} \mathrm{hr}\right)$ & $87(57.3 \%)$ & $65(42.8 \%)$ & $<0.001$ & 1.00 & $80.9 \%(66.7-90.9 \%)$ & $57.2 \%(49.0-65.2 \%)$ \\
\hline & (> $\left.20 \mathrm{~mm} / 1^{\mathrm{st}} \mathrm{hr}\right)$ & $9(19.2 \%)$ & $38(80.9 \%)$ & & $2.11(1.41-3.16)$ & & \\
\hline \multirow[t]{2}{*}{ hsCRP } & $(\leq 8 \mathrm{mg} / \mathrm{L})$ & $84(56.8 \%)$ & $64(43.3 \%)$ & $<0.001$ & 1.00 & $82.2 \%(68.0-92.0 \%)$ & $56.8 \%(48.4-64.9 \%)$ \\
\hline & (> $8 \mathrm{mg} / \mathrm{L})$ & $8(17.8 \%)$ & $37(82.2 \%)$ & & $2.13(1.42-3.20)$ & & \\
\hline \multirow[t]{2}{*}{ IL-6 } & $(\leq 5.9 \mathrm{pg} / \mathrm{ml})$ & $80(61.1 \%)$ & $51(38.9 \%)$ & $<0.001$ & 1.00 & $78.1 \%(62.4-89.4 \%)$ & $61.1 \%(52.2-69.5 \%)$ \\
\hline & (> $5.9 \mathrm{pg} / \mathrm{ml})$ & $9(22.0 \%)$ & $32(78.1 \%)$ & & $2.50(1.60-3.91)$ & & \\
\hline \multirow[t]{2}{*}{ TNFa } & $(\leq 8.1 \mathrm{pg} / \mathrm{ml})$ & $65(63.1 \%)$ & $38(36.9 \%)$ & $<0.001$ & 1.00 & $65.2 \%(52.4-76.5 \%)$ & $63.1 \%(53.0-72.4 \%)$ \\
\hline & $(>8.1 \mathrm{pg} / \mathrm{ml})$ & $23(34.9 \%)$ & $43(65.2 \%)$ & & $2.65(1.69-4.15)$ & & \\
\hline
\end{tabular}

Abbreviation: $H R$ hazard ratio, ESR erythrocyte sedimentation rate, $h s C R P$ high sensitive C-reactive protein, IL-6 interleukin-6, TNFa tumor necrosis factor-a, CI confidence interval

study as an explanatory variable, due to the categories of medication, varied dosages of drugs, and limited sample size. Taking the treatment changes during follow-up visits into consideration, we classified the patients into active and inactive disease at baseline in order to reduce the confounding of treatment to some extents. Doppler ultrasound cannot visualize the thoracic aorta, so it has some limitations in detecting vascular changes in the ascending and thoracic aorta. However, it is more accessible, much cheaper than CTA or MRA, and easier to be performed repeatedly. For those patients with thoracic aorta involvement, we repeated CTA every year or 2 years if there is no evidence of active disease or recurrence, or repeated CTA with shorter interval if there are symptoms and signs suggestive of active disease in this part of aorta (such as persistent back pain or pain at swallow with no other identifiable causes). The concern is that CTA would cause radiological harm particularly for young women with the desire of giving birth and may compromise renal function. Therefore, we would

Table 4 Repeated vascular Doppler imaging results of 354 patients with Takayasu's arteritis in follow-up visits

\begin{tabular}{llll}
\hline & Stable & Improved & Progressed \\
\hline $\mathbf{1 5 2}$ patients in active group at baseline & & & \\
120 patients achieved inactive disease & $72(60.0 \%)$ & $15(12.5 \%)$ & $33(27.5 \%)$ \\
32 patients sustained active disease & $9(28.1 \%)$ & $5(15.6 \%)$ & $18(56.3 \%)$ \\
$\mathbf{2 0 2}$ patients in inactive group at baseline & & & \\
96 patients remained in remission & $53(55.2 \%)$ & $30(31.3 \%)$ & $3(13.5 \%)$ \\
106 patients relapsed & $17(16.0 \%)$ & $3(2.8 \%)$ & $86(81.1 \%)$ \\
\hline
\end{tabular}


repeat CTA when there was clinical symptoms, signs suggesting thoracic aorta involvement, or when the patient needs to have pre-surgery evaluation.

\section{Conclusions}

Elevated level of ESR, CRP, and IL-6 are associated with active disease of TAK in this study. During follow-up, elevated levels of any of these three indices are associated with longer time and lower possibility to achieve inactive disease in patients with active disease in the present visit. Furthermore, elevated levels of ESR, CRP, IL-6, and TNF $\alpha$ are found to be associated with a higher risk for relapse and shorter time to relapse in patients with currently inactive disease. The results of this study suggest that ESR, CRP, IL-6, and TNF $\alpha$ should be tested and monitored for disease activity evaluation during the follow-up of TAK patients. Testing serum TNF $\alpha$ and IL6 is particularly helpful in patients with normal acute phase reactants.

\section{Supplementary Information}

The online version contains supplementary material available at https://doi. org/10.1186/s13075-020-02365-y.

Additional file 1: Supplementary Table S1. The clinical manifestation of 428 patients with Takayasu's arteritis at baseline.

Additional file 2: Supplementary Table S2. 1 Association between ESR, hsCRP, IL-6 and TNFa with disease activity at baseline in univariate logistic regression, excluded patients who were treated with TNFa inhibitors or IL-6 inhibitor and/or patients in pregnancy. Supplementary Table S2.2. Association between ESR, hSCRP, IL-6 and TNFa with disease activity at baseline in univariate logistic regression, excluded patients who were treated with TNFa inhibitors or IL-6 inhibitor.

Additional file 3: Supplementary Table S3. The results of survival analysis by using Kaplan-Meier analysis in 152 patients with Takayasu's arteritis in active group (Fig. 2A-D) at baseline with further follow-up data.

Additional file 4: Supplementary Table S4. The results of survival analysis by using Kaplan-Meier analysis in 202 patients with Takayasu's arteritis in inactive group (Fig. 3A-D) at baseline with further follow-up data.

\section{Abbreviations \\ TAK: Takayasu's arteritis; ESR: Erythrocyte sediment rate; CRP: C-reactive protein; hs-CRP: High-sensitive C-reactive protein; IL-6: Interleukin-6; TNFa: Tumor necrosis factor-a; HR: Hazard ratio; CRSV: Chinese Registry for Systemic Vasculitis; CRDC: Chinese Rheumatism Data Center; ACR: American College of Rheumatology; PUMCH: Peking Union Medical College Hospital; BVAS: Birmingham Vasculitis Activity Score; VDI: Vasculitis Damage Index; CTA: Computed tomography angiography; OR: Odds ratio; Cl: Confidence interval; PGA: Physician global assessment; DEl.Tak: Disease Extent Index- Takayasu; ITAS: Indian Takayasu Activity Score}

\section{Acknowledgements}

Not applicable.

\section{Authors' contributions}

$X T$ and $X Z$ conceived and designed the study. YanhW and $M L$ advised on the design of the study. JL, YahW, YW, YY, and XT collected the data. JL, YanhW, and JZ analyzed and interpreted the patient data. JL, YanhW, and XT were the major contributors in writing the manuscript. The authors read and approved the final manuscript.

\section{Funding}

This work was supported by the National Clinical Research Center for Dermatologic and Immunologic Diseases, the Chinese National Key Research Research and Development Program (grant number 2017YFC0907600, 2008BAI59B02), and the Chinese National High Technology Research and Development Program, Ministry of Science and Technology (grant number 2012AA02A513).

\section{Availability of data and materials}

The datasets used and/or analyzed during the current study are available from the corresponding author on reasonable request.

\section{Ethics approval and consent to participate}

This study was approved by the Institutional Review Board of Peking Union Medical College Hospital, Beijing, China (S-478). Written informed consent was obtained from all participants, and the study was performed in accordance with the Declaration of Helsinki. Personal information was protected and kept anonymous in Chinese Registry of Systemic Vasculitis (CRSV) database

\section{Consent for publication}

Consent for publication has been obtained from all patients.

\section{Competing interests}

The authors declare that they have no competing interests.

\section{Author details}

${ }^{1}$ Department of Rheumatology, Peking Union Medical College Hospital, Peking Union Medical College and Chinese Academy of Medical Sciences, National Clinical Research Center for Dermatologic and Immunologic Diseases (NCRC-DID), Key Laboratory of Rheumatology and Clinical Immunology, Ministry of Education, Beijing 100730, China. ${ }^{2}$ Department of Ultrasound Imaging, Peking Union Medical College Hospital, Peking Union Medical College and Chinese Academy of Medical Sciences, Beijing, China. ${ }^{3}$ Department of Epidemiology and Biostatistics, Institute of Basic Medical Sciences, Chinese Academy of Medical Sciences, School of Basic Medicine, Peking Union Medical College, Beijing, China.

Received: 31 March 2020 Accepted: 3 November 2020

Published online: 10 December 2020

\section{References}

1. Arend WP, Michel BA, Bloch DA, Hunder GG, Calabrese LH, Edworthy SM, et al. The American College of Rheumatology 1990 criteria for the classification of Takayasu arteritis. Arthritis Rheum. 1990;33(8):1129-34.

2. Mason JC. Takayasu arteritis--advances in diagnosis and management. Nat Rev Rheumatol. 2010;6(7):406-15.

3. Park MC, Lee SW, Park YB, Lee SK. Serum cytokine profiles and their correlations with disease activity in Takayasu's arteritis. Rheumatology (Oxford). 2006:45(5):545-8.

4. Savioli B, Abdulahad WH, Brouwer E, Kallenberg CGM, de Souza AWS. Are cytokines and chemokines suitable biomarkers for Takayasu arteritis? Autoimmun Rev. 2017;16(10):1071-8.

5. Arnaud L, Haroche J, Duhaut P, Piette JC, Amoura Z. Pathogenesis of primary large vessel arteritis. Rev Med Interne. 2009;30(7):578-84.

6. Hotchi M. Pathological studies on Takayasu arteritis. Heart Vessels Suppl. 1992:7:11-7.

7. Hellmich B, Agueda A, Monti S, Buttgereit F, de Boysson H, Brouwer E, et al. Update of the EULAR recommendations for the management of large vessel vasculitis. Ann Rheum Dis. 2020;79(1):19-30.

8. Fries JF, Hunder GG, Bloch DA, Michel BA, Arend WP, Calabrese LH, et al. The American College of Rheumatology 1990 criteria for the classification of vasculitis. Summary Arthritis Rheum. 1990;33(8):1135-6.

9. Mukhtyar C, Lee R, Brown D, Carruthers D, Dasgupta B, Dubey S, et al. Modification and validation of the Birmingham Vasculitis Activity Score (version 3). Ann Rheum Dis. 2009;68(12):1827-32.

10. Direskeneli H, Aydin SZ, Merkel PA. Assessment of disease activity and progression in Takayasu's arteritis. Clin Exp Rheumatol. 2011;29(1 Suppl 64):S86-91. 
11. Aydin SZ, Yilmaz N, Akar S, Aksu K, Kamali S, Yucel E, et al. Assessment of disease activity and progression in Takayasu's arteritis with Disease Extent Index-Takayasu. Rheumatology (Oxford). 2010;49(10):1889-93.

12. Misra R, Danda D, Rajappa SM, Ghosh A, Gupta R, Mahendranath KM, et al. Development and initial validation of the Indian Takayasu Clinical Activity Score (ITAS2010). Rheumatology (Oxford). 2013;52(10):1795-801.

13. Kerr GS, Hallahan CW, Giordano J, Leavitt RY, Fauci AS, Rottem M, et al. Takayasu arteritis. Ann Intern Med. 1994;120(11):919-29.

14. Ishihara T, Haraguchi G, Tezuka D, Kamiishi T, Inagaki H, Isobe M. Diagnosis and assessment of Takayasu arteritis by multiple biomarkers. Circ J. 2013; 77(2):477-83.

15. Tamura N, Maejima Y, Tezuka D, Takamura C, Yoshikawa S, Ashikaga T, et al. Profiles of serum cytokine levels in Takayasu arteritis patients: potential utility as biomarkers for monitoring disease activity. J Cardiol. 2017;70(3): 278-85.

16. Comarmond C, Biard L, Lambert M, Mekinian A, Ferfar Y, Kahn JE, et al. Longterm outcomes and prognostic factors of complications in Takayasu arteritis: a multicenter study of 318 patients. Circulation. 2017;136(12):1114-22.

17. Noris M, Daina E, Gamba S, Bonazzola S, Remuzzi G. Interleukin-6 and RANT ES in Takayasu arteritis: a guide for therapeutic decisions? Circulation. 1999; 100(1):55-60.

\section{Publisher's Note}

Springer Nature remains neutral with regard to jurisdictional claims in published maps and institutional affiliations.

Ready to submit your research? Choose BMC and benefit from:

- fast, convenient online submission

- thorough peer review by experienced researchers in your field

- rapid publication on acceptance

- support for research data, including large and complex data types

- gold Open Access which fosters wider collaboration and increased citations

- maximum visibility for your research: over $100 \mathrm{M}$ website views per year

At $\mathrm{BMC}$, research is always in progress.

Learn more biomedcentral.com/submissions 